\title{
In vitro cytotoxicity of silver nanoparticles in primary rat hepatic stellate cells
}

\author{
XIAOJING SUN ${ }^{1}$, ZHIMING WANG ${ }^{1}$, SHENGYONG ZHAI ${ }^{2}$, YINGWEN CHENG ${ }^{3}$, JIE LIU $^{3}$ and BINBIN LIU ${ }^{1}$ \\ ${ }^{1}$ Key Laboratory of Carcinogenesis and Cancer Invasion, Liver Cancer Institute, Zhongshan Hospital, Fudan University, \\ Ministry of Education, Xuhui, Shanghai 200032; ${ }^{2}$ Department of Surgical Oncology, Weifang People's Hospital, \\ Weifang, Shandong 261041, P.R. China; ${ }^{3}$ Department of Chemistry, Duke University, Durham, NC 27708, USA
}

Received March 5, 2013; Accepted August 23, 2013

DOI: $10.3892 / \mathrm{mmr} .2013 .1683$

\begin{abstract}
The number of studies concerning silver nanoparticles (AgNPs) has increased, due in part to their potential uses for biomedical applications. These particles have been demonstrated in the elimination of the hepatitis $B$ virus and the inhibition of the proliferation of various cancer cells in vivo and in vitro. Thus, studies on AgNPs may lead to a more efficacious and safer therapeutic approach for chronic liver injury. Hepatic stellate cells (HSCs) are essential interstitial cells in the liver and are the predominant therapeutic target in hepatic fibrosis and liver cirrhosis; however, the intracellular effects of AgNPs on HSCs remain to be elucidated. The aim of the present study was to investigate the effects of AgNPs on the function and metabolism of HSCs. Various concentrations of AgNPs, with a diameter of 10 or $30-50 \mathrm{~nm}$, were incubated with HSCs. Transmission electron microscopy, flow cytometry, enzyme-linked immunosorbent assays, and apoptosis and proliferation detection kits were used to analyze the effects of AgNPs on cell proliferation and metabolism. These studies demonstrated that AgNPs inhibited the proliferation of HSCs and induced their apoptosis in a size- and dose-dependent manner.
\end{abstract}

\section{Introduction}

Nanomaterials have been widely studied over the past decades, due to their unique chemical and physical properties. At a nanoscale, the properties of materials depend significantly on their particle size and morphology. Silver nanoparticles (AgNPs) are one of the most common commercialized nano-

Correspondence to: Professor Binbin Liu, Key Laboratory of Carcinogenesis and Cancer Invasion, Liver Cancer Insitute, Zhongshan Hospital, Fudan University, Room 309, Building 4, 180 Fenglin Road, Ministry of Education, Xuhui, Shanghai 200032, P.R. China

E-mail: liu.binbin@zs-hospital.sh.cn

Key words: silver nanoparticles, hepatic stellate cells, cytotoxicity, size dependent, dose dependent materials used in key biological and medical studies for applications such as antimicrobial agents, drug and gene delivery vehicles and biosensors. $(1,2)$

AgNPs have been demonstrated to effectively inhibit the replication of hepatitis B virus (HBV) (3). Injection of 10 and $50 \mathrm{~nm}$ diameter AgNPs into patients with HBV reduced the quantity of $\mathrm{HBV}$ by $40 \%$ within $10 \mathrm{~min}$ and by $90 \%$ after $1 \mathrm{~h}$ (3). In addition, these nanoparticles have been used to target cancer cells with the controlled uptake into specific cellular compartments. Specifically, nanoparticles may be delivered to specific tissues and subcellular compartments or to malignant cells in circulation via a combination of antibody-based targeting of ligands, changing of surface charge and material composition (4). These studies provided the basis for the prevention and cure of HBV infection and also provided a reference for chronic liver disease, such as hepatic fibrosis.

In the clinic, conventional anti-fibrotic treatments and chemotherapy are of limited success in chronic liver injury, predominantly due to non-specific drug effects and the development of drug tolerance. Thus, AgNPs may provide a more efficacious and safer therapeutic approach. The possibility of this approach is important for hepatic stellate cells (HSCs), which are considered to be targets for the therapy of hepatic fibrosis and liver cirrhosis. Thus, investigation of the cytotoxicity of AgNPs in HSCs may be useful in determining their potential use in clinical applications.

In this study, the cytotoxicity of polyvinylpyrrolidone (PVP)-coated AgNPs in primary HSCs derived from fresh rat livers was determined. The biological responses of HSCs, such as the morphological changes in subcellular structure, proliferation, apoptosis, cell movement and cytokine secretion, were determined following the treatment of cells with AgNPs. Different particle sizes and concentrations of AgNPs were also used to study their effects on the cytotoxicity of AgNPs.

\section{Materials and methods}

Preparation and characterization of AgNPs. PVP-coated AgNPs with diameters of 10 and 30-50 nm were received in solution from Dr Jie Liu (Duke University, Durham, NC, USA). The size, morphology and dispersion of the nanoparticles were characterized using a Tecnai ${ }^{\mathrm{TM}}$ G2 Twin Transmission 
Electron Microscope (FEI, Hillsboro, OR, USA) and dynamic light scattering (Compact Goniometer System 3; ALV-GmbH, Langen, Germany). The $\zeta$ potential was determined using a Zetasizer Nano ZS (Malvern Instruments, Malrem, UK). X-ray diffraction experiments were performed on powder samples and were analyzed using an X'Pert PRO MRD HR diffractometer with a $\mathrm{Cu} \mathrm{K} \alpha$ radiation $(1.5405 \AA$ ) at $5 \mathrm{kV}$ and $40 \mathrm{~mA}$ (PANalytical, Almelo, Netherlands). X-ray photoelectron spectroscopy (Kratos Analytical Inc., Chestnut Ridge, Spring Valley, NY, USA) was used to determine the composition of the surface coating.

Isolation and culture of rat HSCs. HSCs were isolated from the livers of six normal Buffalo rats aquired from the Chinese Academy of Sciences (Shanghai, China) using the improved Friedman method (5). The study was approved by the Ethics Committee of Fudan University, Shanghai, China. Following isolation, the cells were cultured in Dulbecco's modified Eagle's medium, supplemented with $10 \%$ fetal bovine serum, in a humidified incubator at $37^{\circ} \mathrm{C}$ with $5 \% \mathrm{CO}_{2}$. The morphology and features of HSCs were observed with light and fluorescence microscopes. Cell purity was determined by immunofluorescence staining for desmin and smooth muscle actin (SMA) using anti- $\alpha$-SMA antibodies from Sigma-Aldrich (St. Louis, MO, USA). Cells were counterstained with fluorescein isothiocyanate (FITC)-conjugated goat anti-mouse IgG (Becton-Dickinson, New York, NY, USA) and nuclei were stained with DAPI. The purity of HSCs (\%) was calculated as the number of desmin-positive (or $\alpha$-SMA-positive) cells divided by the total number of cells multiplied by $100 \%$. HSC activation was determined by $\alpha$-SMA immunofluorescence staining.

Transmission electron microscopy observation. Transmission electron microscopy (TEM; CM12; Philips, Amsterdam, Netherlands) was used to examine the morphology of HSCs. HSCs were seeded in 100-mm tissue culture dishes, cultured for 2 days and treated with different concentrations of AgNPs for $24 \mathrm{~h}$.

Assessment of in vitro cytotoxicity. Cell proliferation was evaluated with a Cell Counting Kit-8 (CCK-8; Becton Dickinson). Approximately $5 \times 10^{3}$ cells were plated in each well of a 96-well plate. The cells were divided into seven groups. Group 1 served as a blank control; groups 2, 3, and 4 were treated with 30-50 nm AgNPs at 20, 100 and $250 \mu \mathrm{g} / \mathrm{ml}$, respectively; and groups 5, 6 and 7 were treated with $10 \mathrm{~nm} \mathrm{AgNPs}$ at 20, 100 and $250 \mu \mathrm{g} / \mathrm{ml}$, respectively. Following incubation for $96 \mathrm{~h}, 10 \mu \mathrm{l}$ WST-8 was added to the wells and the absorbance at $450 \mathrm{~nm}$ was determined using a microplate reader (Thermo Fisher Scientific, Inc., Waltham, MA, USA). The aforementioned samples were analyzed for $24 \mathrm{~h}$.

Apoptosis detection. The FITC-Annexin V and propidium iodide (PI) double staining method was used to detect apoptosis induced by AgNPs. Freshly isolated HSCs were cultured in 96-well plates for 2 days and were divided into seven groups as described previously for the cytotoxicity studies. Following $24 \mathrm{~h}$ of nanoparticle treatment, the cells were washed with cold phosphate-buffered saline $\left(4^{\circ} \mathrm{C}\right)$ and stained using the
FITC-Annexin V Apoptosis Detection kit (ExCell Biology, Inc., Shanghai, China). The stained cells were analyzed by flow cytometry by a trained laboratory technician according to the experimental protocol.

Analysis of AgNP acute toxicity by a lactase dehydrogenase ( $L D H)$ activity assay. Rat LDH kits (Sigma-Aldrich) were used to conduct the LDH leakage assay. Two days following seeding in 24-well plates, the cells were divided into two groups. One group was treated with AgNPs of different diameters and different concentrations, and the other group was treated as a control without nanoparticle treatment. The two groups were incubated at $37^{\circ} \mathrm{C}$ with $5 \% \mathrm{CO}_{2}$ for $4 \mathrm{~h}$ according to the manufacturer's instructions. The cell culture medium $(50 \mu \mathrm{l})$ was collected from each well and analyzed using a spectrometer (UV-Vis-NIR spectrophotometer; Agilent, Santa Clara, CA, USA).

Cell IQ. The cell biological behaviors, including the total cell number, number of dead cells and cell movement, were measured using a real-time cell-monitoring system with a cell-culturing platform (Cell-IQ; Chip-Man Technologies, Tampere, Finland). HSCs were cultured in the Cell-IQ system in 24-well plates $\left(1 \times 10^{4}\right.$ cells/well) for $72 \mathrm{~h}$. Cells were divided into five groups with two wells per group. One group served as a blank control and the remaining four groups were treated with large and small AgNPs at 20 and $100 \mu \mathrm{g} / \mathrm{ml}$, respectively.

Cytokine detection. The HSCs were adjusted to a concentration of $5 \times 10^{5}$ cells $/ \mathrm{ml}$ and cultured in 24 -well plates. The 24 wells were divided into three groups; one group served as the blank control and the other two groups were treated with AgNPs (with diameters of either 10 or $30-50 \mathrm{~nm}$ ) at a concentration of $20 \mu \mathrm{g} / \mathrm{ml}$. The conditioned medium was collected after 2 days and the quantity of hepatocyte growth factor (HGF), interleukin (IL)-6, transforming growth factor (TGF)- $\beta 1$, tumor necrosis factor (TNF)- $\alpha$, matrix metallopeptidase (MMP)-2 and MMP-9 in the serum-free HSC-conditioned medium was quantified using an enzyme-linked immunosorbent assay (ELISA) kit (ExCell Biology, Inc.) according to the manufacturer's instructions.

Statistical analysis. All experiments were repeated at least three times, and the data are presented as the mean \pm standard deviation. Student's t-test was performed to determine the statistical significance of the difference between untreated cells (blank control) and cells treated with AgNPs. P<0.05 was considered to indicate a statistically significant difference.

\section{Results}

Characterization of AgNPs. TEM images of the AgNPs used in this study are shown in Fig. 1. The nanoparticles were all roughly spherical in shape, although a few aggregates were also observed. The diameters of these two types of AgNPs were $30 \pm 10$ and $80 \pm 40 \mathrm{~nm}$ as determined by TEM. The nanoparticles consisted of $\mathrm{C}, \mathrm{O}, \mathrm{N}$ and $\mathrm{Ag}$, were metallic silver with a face-centered cubic lattice and were polydispersed. The broad absorption at wavelengths $>500 \mathrm{~nm}$ as 
Table I. Characterization of silver nanoparticles.

Silver nanoparticle

Property

\begin{tabular}{cc}
\hline Small & Large \\
\hline 10 & $30-50$ \\
$30 \pm 10$ & $80 \pm 40$ \\
$28.6 \pm 0.61$ & $58.74 \pm 1.49$ \\
(PDI: 0.395$)$ & (PDI: 0.491$)$ \\
$-24.5 \pm 17.2$ & $-28.6 \pm 5.54$ \\
85.2 & 87.3
\end{tabular}

Nominal diameter (nm)

TEM diameter (nm)

Hydrodynamic radius using DLS (nm)

$28.6 \pm 0.61$

$58.74 \pm 1.49$

$\zeta$ Potential $(\mathrm{mV})$

85.2

87.3

Data are presented as the mean \pm SD. TEM, transmission electron microscopy; DLS, dynamic light scattering; PDI, polydispersity index; ICP-MS, inductively coupled plasma mass spectrometer (Agilent, Santa Clara, CA, USA).

A

a

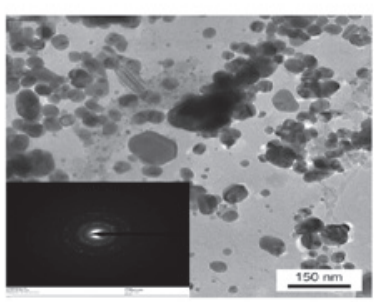

B

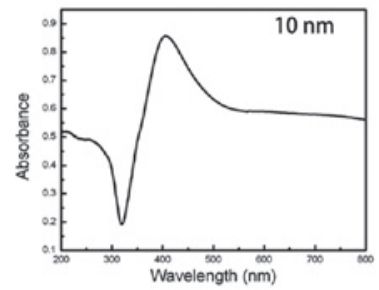

C

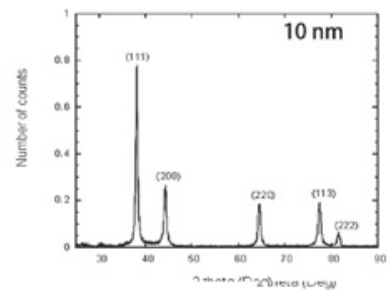

D

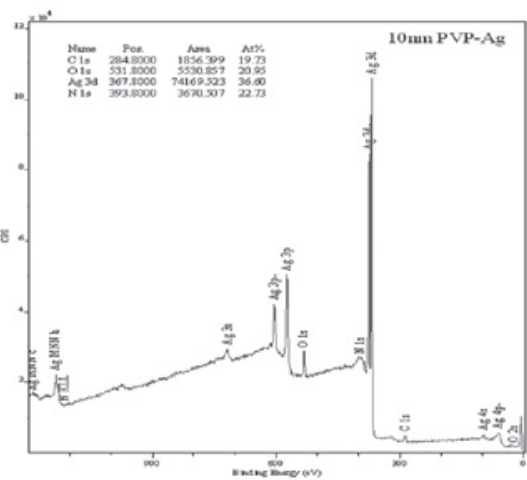

b
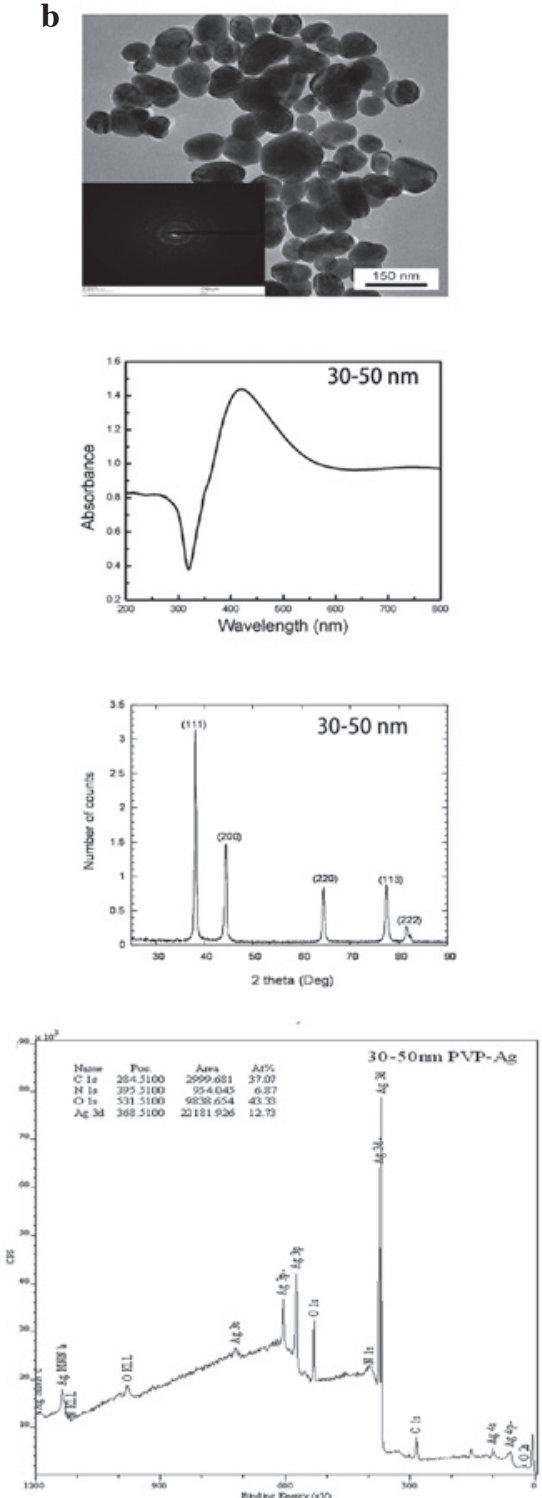

Figure 1 Characterization of silver nanoparticles (AgNPs). (Aa) Transmission electron microscopy (TEM) images for small AgNPs. The mean diameter was $30 \pm 10 \mathrm{~nm}$. (Ab) TEM images for large AgNPs. The mean diameter was $80 \pm 40 \mathrm{~nm}$. (B) Ultraviolet-visible (UV-Vis) absorption spectra of both AgNPs were acquired with a Cary 500 scan UV-Vis-NIR spectrophotometer. The material was polydispersed, and the broad absorption $>500 \mathrm{~nm}$ indicated the possible existence of large aggregates. (C) The characteristic X-ray diffraction pattern of the two sizes of AgNPs used was obtained using a X'Pert PRO MRD HR diffractometer. The AgNPs appeared as metallic silver with a face-centered cubic lattice. (D) Axis Ultra X-ray photoelectron spectroscopy was used to evaluate the elemental composition in the two sizes of nanoparticles with respect to $\mathrm{C}, \mathrm{O}, \mathrm{N}$ and $\mathrm{Ag}$, using anode mode and aluminum monochromatic energy sources. 
A

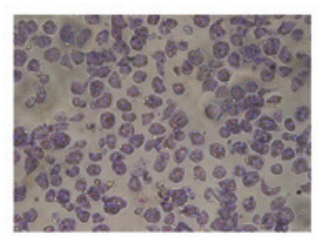

$\mathbf{E}$

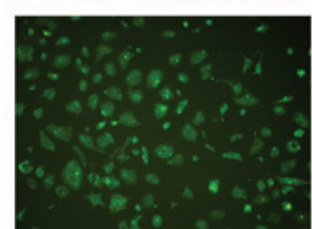

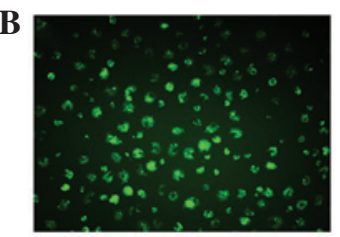

F

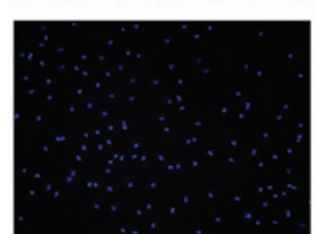

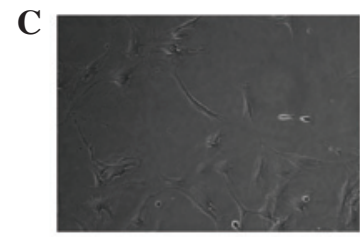

G

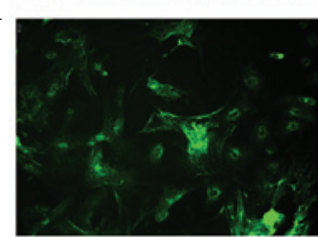

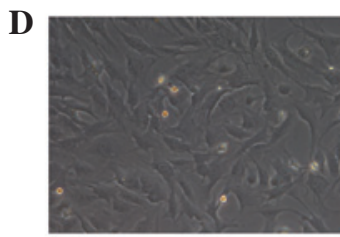

$\mathbf{H}$

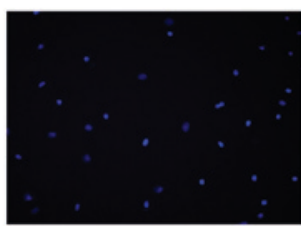

Figure 2. Characterization of hepatic stellate cells (HSCs) isolated from rat livers. To identify the HSCs, the newly isolated cells were cultured for 2 days (quiescent HSCs), 4 days and 7 days (activated HSCs). (A) Giemsa staining for the quiescent HSCs. The typical light microscopic appearance of lipid droplets is shown. (B) Fluorescence microscope image depicts the cyan vitamin A auto-fluorescence of quiescent HSCs. (C) Following culture for 4 days, HSCs exhibited a distinct star-like morphology in optical micrographs. (D) Activated HSCs demonstrated fibroblast-like morphology in optical micrographs. (E) Immunofluorescence analysis of desmin expression in qHSCs. Immunofluorescence staining for desmin was performed using an anti-desmin antibody. (G) $\alpha$-smooth muscle actin (SMA)-positive activated HSCs. Immunofluorescence staining for $\alpha$-SMA was performed using an anti- $\alpha$-SMA antibody. (F) Desmin and $(\mathrm{H}) \alpha$-SMA staining for HSC nuclei.
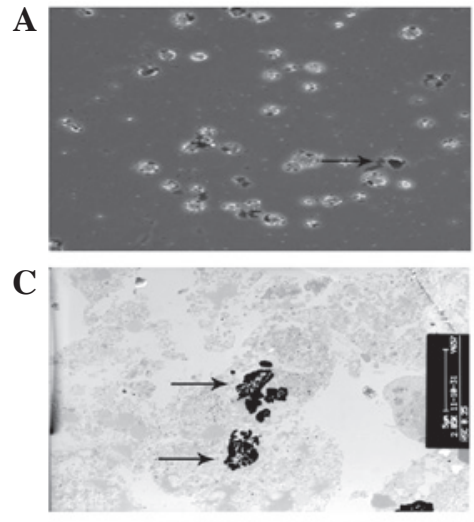

E

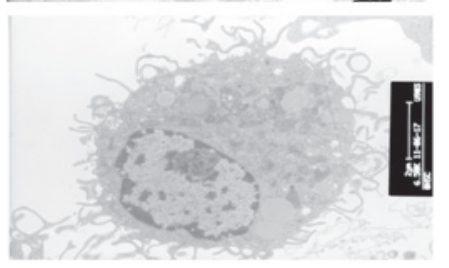

B

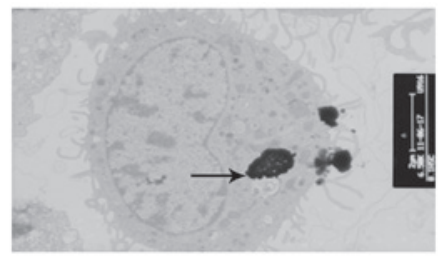

D

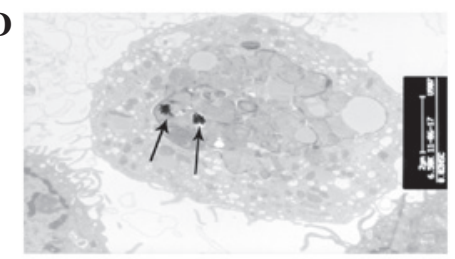

F

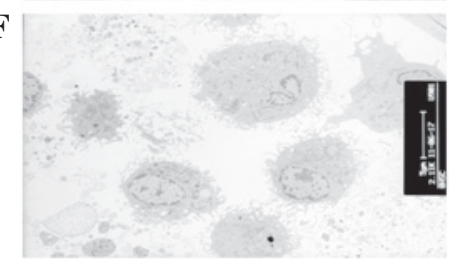

Figure 3. The morphology of hepatic stellate cells (HSCs) incubated with varying concentrations of two different sizes of silver nanoparticles (AgNPs; arrows) indicated that apoptosis or necrosis was induced by the AgNPs. (A) Optical micrographs of HSCs treated with smaller AgNPs at $100 \mu \mathrm{g} / \mathrm{ml}$ for $24 \mathrm{~h}$. AgNPs accumulated on the cell surface and between cells. (B) Transmission electron microscopy (TEM) images of HSCs treated with larger AgNPs at $100 \mu \mathrm{g} / \mathrm{ml}$ for $24 \mathrm{~h}$ demonstrated that the AgNPs aggregated in the cytoplasm. (C) HSCs incubated with $250 \mu \mathrm{g} / \mathrm{ml}$ of small nanoparticles for 7 days exhibited karyorrhexis, karyolysis and karyotheca disintegration in TEM images. (D) HSCs treated with $20 \mu \mathrm{g} / \mathrm{ml}$ of large nanoparticles for 2 days exhibited mitochondrial swelling by TEM. (E and F) HSCs without any treatment exhibited a normal cell structure under TEM.

shown in the ultraviolet-visible absorption spectra also indicated the possible existence of aggregates (Fig. 1). The results of the other characterization experiments are summarized in Table I.

Identification of HSCs. Approximately $2 \times 10^{7} \mathrm{HSCs}$ were isolated from each rat. The morphology of freshly isolated HSCs exhibited no obvious features (Fig. 2A). Abundant lipid droplets were observed with light microscopy and the cyan vitamin A autofluorescence was excited at $328 \mathrm{~nm}$, as observed with fluorescence microscopy (Fig. 2B). However, activated HSCs demonstrated star-like morphology (Fig. 2C) and developed into fibroblast-like cells 7 days following isola- tion (Fig. 2D). More than 95\% of the HSCs showed positive desmin staining, indicating that the population consisted of pure HSCs (Fig. 2E and F). In addition, $>95 \%$ of the HSCs were positive for $\alpha$-SMA, also suggesting a pure population (Fig. 2G and $\mathrm{H}$ ).

Ultrastructural characteristics of AgNP-treated HSCs. According to the TEM analysis, AgNPs were rapidly internalized by HSCs, although the majority of the nanoparticles were observed on the cell surface and between the cells (Fig. 3A), The TEM images of AgNP-treated cells demonstrated the presence of electron-dense, aggregated regions, which were thought to be AgNPs in the cytoplasm (Fig. 3B). Moreover, 
A

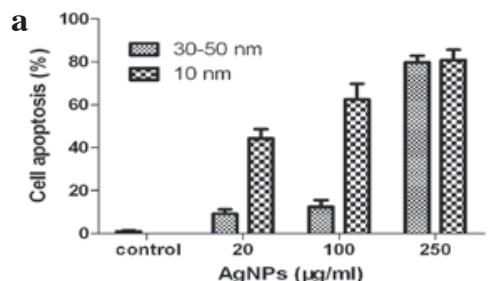

B

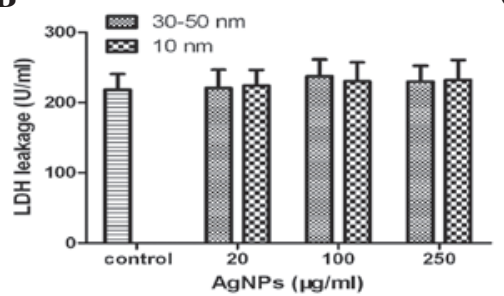

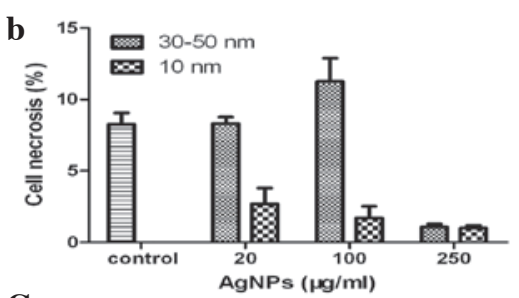

C

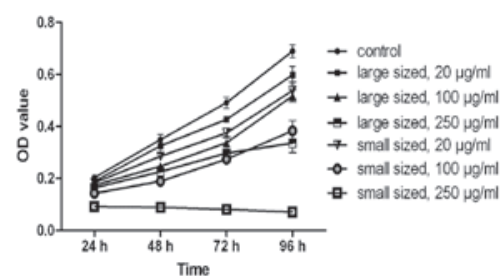

Figure 4. In vitro cytotoxicity of silver nanoparticle (AgNP)-treated hepatic stellate cells (HSCs). AgNPs induced the (Aa) apoptosis and (Ab) necrosis of HSCs. HSCs were incubated with the two sizes of nanoparticles at various concentrations for $24 \mathrm{~h}$. The AgNPs induced apoptosis or necrosis of HSCs in a size- and dose-dependent manner $(\mathrm{P}<0.05)$. (B) Acute toxicity of AgNPs on HSCs following incubation for $4 \mathrm{~h}$. The quantity of lactose dehydrogenase (LDH) released into the medium was measured by spectrophotometric analysis. Statistical analysis indicated no significant difference of the treated groups compared with the control group. (C) Cytotoxicity of AgNPs as determined using a CCK-8 kit. HSCs were treated with AgNPs of two different sizes (large and small) at varying concentrations as indicated for different lengths of time $(24,48,72$ and $96 \mathrm{~h})$ and cell toxicity was size- and dose-dependent. Statistical analysis demonstrated significant differences between the treated and untreated control cells $(\mathrm{P}<0.05)$.

A

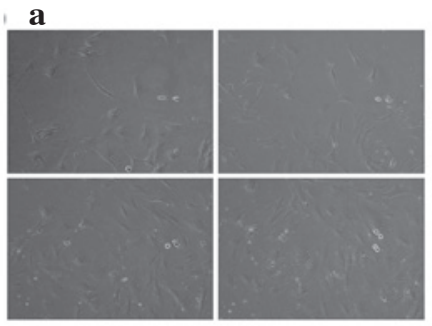

B

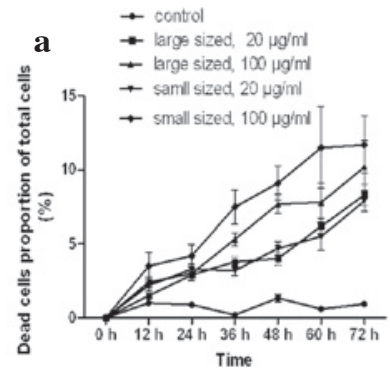

b

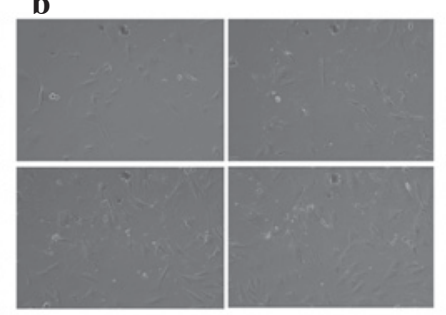

b

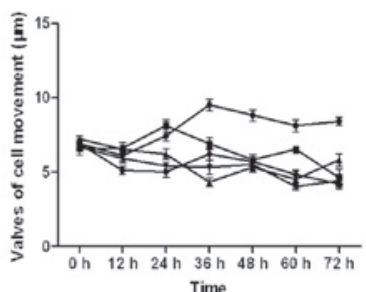

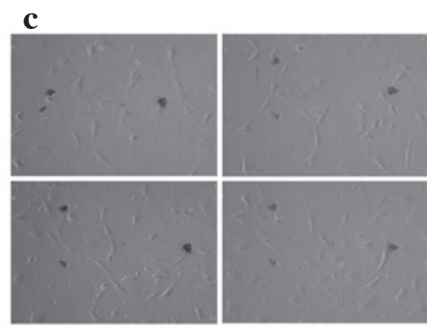

c

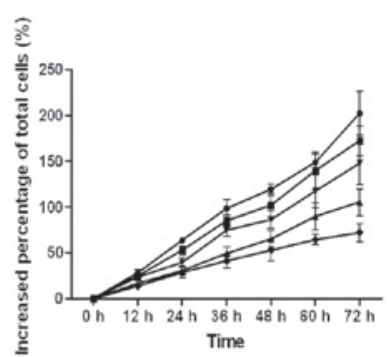

Figure 5. Inhibitory effects of silver nanoparticles (AgNPs) on the bio-behaviors of hepatic stellate cells (HSCs). Following culture of HSCs with or without AgNPs of the two sizes at either $20 \mu \mathrm{g} / \mathrm{ml}$ or $100 \mu \mathrm{g} / \mathrm{ml}$ for $72 \mathrm{~h}$, the targets were measured by a real-time cell-monitoring system. Images were captured at 5 -min intervals for $12 \mathrm{~h}$. Data are presented as the mean $\pm \mathrm{SD}$, and each group comprises eight measurements. (Aa) Control cells (untreated). (Ab) Cells treated with large AgNPs at $100 \mu \mathrm{g} / \mathrm{ml}$. (Ac) Cells treated with small AgNPs at $100 \mu \mathrm{g} / \mathrm{ml}$. (Ba) Dead cells as a proportion (\%) of total cells were calculated according to the following formula: Rate $(\%)=$ number of dead cells/number of primary seeded cells x 100. (Bb) Cell movement was calculated as the sum of the distance of movement of each cell between two images (5-min intervals) / number of primary seeded cells. (Bc) Increased percentage (\%) of total cell number was calculated as the following: Rate $(\%)=$ (value at each time point-value of primary seeding cells) / value of primary seeding cells x 100 .

AgNP-treated HSCs exhibited karyolysis and ruptured cell membranes (Fig. 3C). The presence of large vacuoles and the swelling of the mitochondria indicated the destruction of organelles in the treated cells (Fig. 3D).

Apoptosis detection. To investigate the mechanism of cell death induced by AgNPs, the treated cells were stained with FITC-Annexin V and PI. The smaller AgNPs exhibited a greater ability to induce apoptosis and necrosis in the HSCs than the larger AgNPs (Fig. 4A). Morever, treatment of HSCs with higher concentrations of AgNPs induced greater rates of apoptosis and necrosis than the lower concentrations of AgNPs. Cell death was primarily due to a size-dependent increase in apoptosis induced by the smaller AgNPs. In addition, the large and small AgNPs induced greater apoptosis than necrosis in treated HSCs (Fig. 4Aa). Notably, AgNPs induced the greatest apoptosis and the least necrosis at the highest concentration tested $(250 \mu \mathrm{g} / \mathrm{ml})$. 

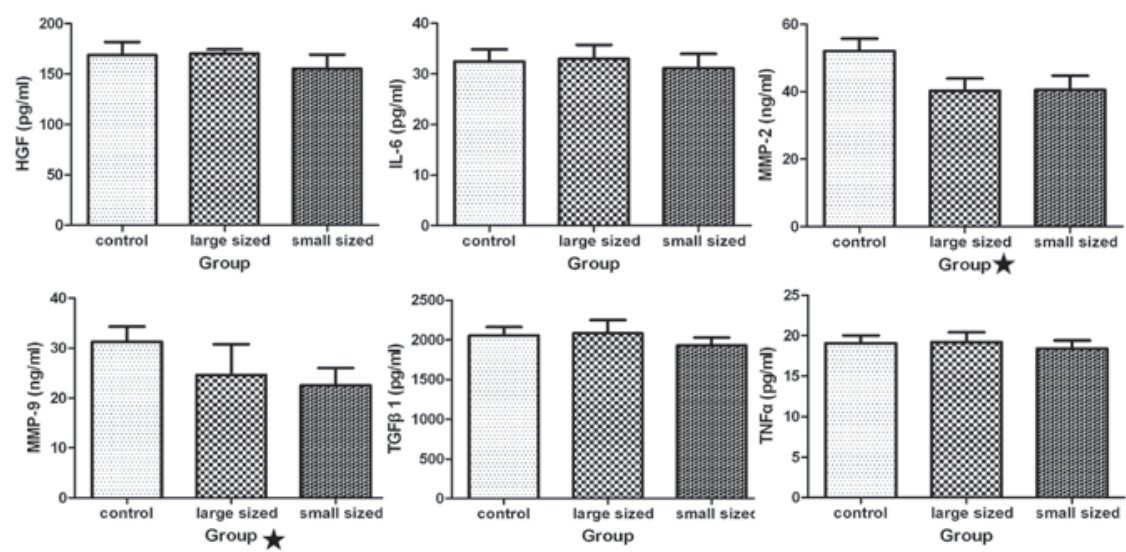

Figure 6. Influence of silver nanoparticles (AgNPs) on cytokine production. Following incubation of hepatic stellate cells (HSCs) with AgNPs of different sizes at $0.2 \mathrm{mg} / \mathrm{ml}$ for $48 \mathrm{~h}$, the levels of various cytokines [hepatocyte growth factor (HGF), interleukin (IL)- 6 , transforming growth factor (TGF)- $\beta 1$, tumor necrosis factor (TNF)- $\alpha$, matrix metallopeptidase (MMP-2) and MMP-9] in the medium were measured using an enzyme-linked immunosorbent assay kit. Statistical analysis showed that the levels of MMP-2 and -9 were significantly different in cultures of cells incubated with AgNPs as compared with the medium from the untreated cells $(\mathrm{P}<0.05)$, while the other four groups were not significantly different compared with the control group (P>0.05). Control, HSCs treated without AgNPs; large sized, HSCs treated with AgNPs, with a diameter of 30-50 nm; small sized, HSCs treated with AgNPs, with a diameter of 10 nm.

Acute HSC cytotoxicity and AgNPs. The effect of AgNPs on the plasma membrane was not statistically significant at any of the concentrations tested (P>0.05; Fig. 4B). Thus, the HSC death was not able to be correlated with the acute toxicity of AgNPs. The aforementioned results also indicated the primary cause of HSC death was not due to the acute toxicity of AgNPs.

In vitro cytotoxicity of AgNPs. The time-dependent cytotoxicity of AgNPs was assessed using the CCK-8 assay (Fig. 4C). HSC growth was arrested in the presence of the two sizes of nanoparticles at various concentrations. The influence of particle size and concentration on cell proliferation was determined. Treatment of cells with $100 \mu \mathrm{g} / \mathrm{ml} \mathrm{AgNPs}$ resulted in a decrease in cell proliferation after $96 \mathrm{~h}$ of exposure. These effects were also dependent on the AgNP diameter size. In particular, the smaller nanoparticles induced a greater decrease in cell viability than larger ones. Furthermore, in cases of treatment with identically sized nanoparticles, there was a greater inhibitory effect with an increased concentration.

Inhibitory effects of AgNPs on cell biological behaviors. Dynamic alterations of the total cell number, number of dead cells and cell movement were measured using a Cell-IQ culturing platform. The increased percentage of the total cell number was significantly lower following incubation with AgNPs, than in untreated cells $(\mathrm{P}<0.05)$. The inhibitory effects of AgNPs were dependent on the size and dose (Fig. 5Bc). The number of dead cells as a percentage of total cells in the control group was significantly lower than that in the groups treated with AgNPs $(\mathrm{P}<0.05)$, and the effect was dose- and size-dependent (Fig. 5Ba). Similarly, the movement of AgNP-treated HSCs was inhibited $(\mathrm{P}<0.05)$. The diameter of the AgNPs, however, was not significantly correlated with cell migration at high concentrations $(\mathrm{P}>0.05)$.

Effect of AgNPs on various cytokines. Analysis of cytokine levels following treatment of HSCs with AgNPs was performed using ELISA. Production of HGF, IL-6, TGF- $\beta 1$, and TNF- $\alpha$ was not significantly different between the treated and untreated groups (Fig. 6). The cells treated with AgNPs produced less MMP-2 and MMP-9 than the control cells $(\mathrm{P}<0.05)$.

\section{Discussion}

Previously, AgNPs have been studied as a result of their ability to inhibit HBV and their function in drug delivery and targeting, which may lead to their application for treating liver diseases $(1,6,7)$. With such considerable interest in the development of AgNPs for medical applications, there is concern regarding their cytotoxicity and its potential mechanism of action. Consequently, this study investigated the cytotoxic effects of AgNPs on HSCs. The study demonstrated three key results: i) the AgNPs exerted a strong negative effect on HSCs, even at low concentration; ii) the particle size of the AgNPs affected the cytotoxicity, as the smaller nanoparticles exerted larger effects on cell bioactivity than the larger nanoparticles; and iii) AgNPs altered the secretion of cytokines by HSCs, which may further affect the microenvironment of HSC activation.

Previous studies have demonstrated the impact of nanoparticle size on cellular uptake and consequent cytotoxicity $(8,9)$. In the present study, the cytotoxic effects of AgNPs, according to their size and concentration, were determined by the investigation of AGNPs of two distinct sizes at various concentrations. The results of the CCK-8 assay demonstrated that the cytotoxic effects exerted by AgNPs on HSCs are sizeand dose-dependent. Smaller AgNPs induced greater HSC cytotoxicity than the larger particles, and at higher concentrations, these nanoparticles induced greater apoptosis. The death of HSCs was not due to acute toxicity, according to our studies, which confirmed the relatively safe use of HSCs in vivo. Morever, the mechanism of cell death induced by AgNPs was analyzed and it was demonstrated that the cell death of AgNP-treated HSCs was predominantly due to apoptosis. The induced HSC apoptosis primarily occurred in cells treated with the smaller nanoparticles and the apoptotic behavior was dose dependent. To enter the cell nucleus, nanoparticles must be 
small enough to pass through the nuclear pore complex on the nuclear membrane. The size of AgNPs is therefore significant in cellular uptake, and thus may impact their bioactivity (10). As a result, consideration of the particle size is required in the design of nanoparticles for biomedical uses.

The changes in HSC morphology were studied using phase contrast microscopy and TEM prior to and following incubation with two sizes of AgNPs at various concentrations. These studies indicated that AgNPs induced HSCs apoptosis or necrosis. The ultrastructural characteristics of apoptosis are cell shrinkage, karyopyknosis, karyorrhexis and karyolysis. Karyorrhexis is a type of destructive fragmentation of the nucleus and is preceded by pyknosis and followed by karyolysis (11). This fragmentation appeared predominantly in HSCs treated with $250 \mu \mathrm{g} / \mathrm{ml}$ of smaller AgNPs (Fig. 3C). Karyotheca disintegration followed by karyolysis is the complete dissolution of the chromatin matter of a dying cell. Cell membrane rupture and the eventual fragmentation of the cell into apoptotic bodies that are engulfed by neighboring cells or phagocytes was observed in HSCs treated with AgNPs (12). The cellular uptake of smaller nanoparticles may be easier than the uptake of larger nanoparticles, although this hypothesis requires additional confirmation by quantitative analysis. Necrosis, however, occurs when the cell is sacrificed and is characterized by cell swelling, cell organelle swelling and the formation of microvesicles (Fig. 3D). This cellular swelling may be accompanied by an increase in intracellular pressure that leads to the breakage of the cell membrane, which allows leakage of the cytoplasm into the intercellular space (13).

The mitochondrial swelling observed following incubation of HSCs with AgNPs indicated that the AgNPs in the cytoplasm primarily reside in the mitochondria, affecting their function and consequently exerting effects on cell metabolism. Mitochondria are important signaling centers during apoptosis, and the loss of mitochondrial integrity is induced and inhibited by numerous regulators of apoptosis (14). For example, Bcl-2 prevents the opening of the mitochondrial membrane pore, whereas Bax accelerates this opening (15). AgNPs have been demonstrated to activate the intrinsic apoptotic pathway, which is characterized by the modulation of Bax and Bcl-2 expression, disruption of the mitochondrial membrane potential, and cytochrome $c$ release from the mitochondria (16). During the apoptotic process, the mitochondrial membrane pores are opened and the mitochondrial membrane potential is disrupted (17). Loss of mitochondrial membrane potential is followed by cytochrome $c$ release from the mitochondria, resulting in the activation of caspase- 9 and -3 .

Activated HSCs are important in chronic liver disease through the production of various cytokines. The ELISA assay confirmed that AgNPs inhibited the production of MMP-2 and -9 , which are known to be crucial in chronic liver injury. During hepatic inflammation and hepatocellular necrosis, MMP-2 and -9 are predominantly produced by myofibroblasts, which transdifferentiate from activated HSCs (18). In chronic liver disease, increases in MMP-2 and -9 are associated with fibrosis and the development of cirrhosis through altered matrix production and degradation (19). In addition, the overexpression of MMP-2 and -9 has been detected in hepatocellular carcinoma (HCC) (20). Moreover, the overexpression of MMP-2 and -9 in HCC tissues is correlated with liver cirrhosis, capsular invasion, the presence of intrahepatic metastasis, vascular invasion and higher tumor-node-metastasis stage (20). Therefore, the inhibitory effects of AgNPs on certain cytokines may destroy the microenvironment in hepatic fibrosis and cirrhosis; however, the detailed mechanisms require further investigation.

In conclusion, the cytotoxicity of AgNPs was investigated by various biochemical approaches and was particle size- and dose-dependent. Morphology alterations may be an indication of metabolic and structural disturbances of HSCs caused by AgNPs. Thus, it was concluded that these alterations were size-dependent, as smaller particles induced greater cellular damage at the same concentrations. The results of the LDH assay confirmed that the necrosis or apoptosis of HSCs was not due to the acute toxicity of AgNPs but due to the effects of the nanoparticles on the physiological activities of the cells. These findings may illustrate the relative safety of AgNP application to the human body. AgNPs also affected the HSC cytokine secretion, as demonstrated by the inhibitory effects of AgNPs on the production of MMP-2 and -9. These results suggested that AgNPs may be used in the treatment of hepatic fibrosis, including HCC; however, the molecular mechanisms of nanoparticle cytotoxicity remain unclear. Therefore, further studies are required to elucidate particle-cell interactions and the metabolic and immunological responses activated in HSCs in the presence of AgNPs.

\section{Acknowledgements}

This study was conducted at Duke University and was supported by the National Science Foundation (NSF) and the Environmental Protection Agency (EPA) under NSF Cooperative Agreement EF-0830093, Center for the Environmental Implications of NanoTechnology. Any opinions, findings, conclusions or recommendations expressed in this material are those of the author(s) and do not necessarily reflect the views of the NSF or the EPA. This study has not been subjected to EPA review and no official endorsement should be inferred.

\section{References}

1. AshaRani PV, Low Kah Mun G, Hande MP and Valiyaveettil S: Cytotoxicity and genotoxicity of silver nanoparticles in human cells. ACS Nano 3: 279-290, 2009.

2. Xu X, Yang Q, Bai J, Lu T, Li Y and Jing X: Fabrication of biodegradable electrospun poly(L-lactide-co-glycolide) fibers with antimicrobial nanosilver particles. J Nanosci Nanotechno 8: 5066-5070, 2008.

3. Lu L, Sun RW, Chen R, Hui CK, Ho CM, Luk JM, Lau GK and Che CM: Silver nanoparticles inhibit hepatitis B virus replication. Antivir Ther 13: 253-262, 2008.

4. Schroeder S, Heller DA, Winslow MM, et al. Treating metastatic cancer with nanotechnology. Nat Rev Cancer 12: 39-50, 2011.

5. Weiskirchen R and Gressner AM: Isolation and culture of hepatic stellate cells. Methods Mol Med 117: 99-113, 2005.

6. Lara HH, Ayala-Nuñez NV and Ixtepan-Turrent L and Rodriguez-Padilla C: Mode of antiviral action of silver nanoparticles against HIV-1. J Nanobiotechnology 8: 1-10, 2010.

7. Gopinath P, Gogoi SK, Chattopadhyay A and Ghosh SS: Implications of silver nanoparticle induced cell apoptosis for in vitro gene therapy. Nanotechnology 19: 104-113, 2008.

8. Yuan Y, Liu C, Qian J, Wang J and Zhang Y: Size-mediated cytotoxicity and apoptosis of hydroxyapatite nanoparticles in human hepatoma HepG2 cells. Biomaterials 31: 730-740, 2010. 
9. Abdelhalim MA and Jarrar BM: Gold nanoparticles induced cloudy swelling to hydropic degeneration, cytoplasmic hyaline vacuolation, polymorphism, binucleation, karyopyknosis, karyolysis, karyorrhexis and necrosis in the liver. Lipids Health Dis 10: 166, 2011.

10. Li Y, Tian X, Lu Z, Yang C, Yang G, Zhou X, Yao H, Zhu Z, $\mathrm{Xi} \mathrm{Z}$ and Yang $\mathrm{X}$ : Mechanism for alpha- $\mathrm{MnO}_{2}$ nanowire-induced cytotoxicity in Hela cells. J Nanosci Nanotechnol 10: 397-404, 2010.

11. Zamzami N and Kroemer G: Condensed matter in cell death. Nature 401: 127-128, 1999.

12. Urne AG and Vaux DL: Molecular and clinical aspects of apoptosis. Pharmacol Ther 72: 37-50, 1996.

13. Schrand AM, Rahman MF, Hussain SM, Schlager JJ, Smith DA and Syed AF: Metal-based nanoparticles and their toxicity assessment. Wiley Interdiscip Rev Nanomed Nanobiotechnol 2: 544-568, 2010

14. Green DR and Reed JC: Mitochondria and apoptosis. Science 281: 1309-1312, 1998.

15. Zamzami N, Marchetti P, Castedo M, Zanin C, Vayssière JL, Petit PX and Kroemer G: Reduction in mitochondrial potential constitutes an early irreversible step of programmed lymphocyte death in vivo. J Exp Med 181: 1661-1672, 1995.
16. Piao MJ, Kang KA, Lee IK, Kim HS, Kim S, Choi JY, Choi J and Hyun JW: Silver nanoparticles induce oxidative cell damage in human liver cells through inhibition of reduced glutathione and induction of mitochondria-involved apoptosis. Toxicol Lett 201: 92-100, 2011.

17. Kroemer G, Zamzami N and Susin SA: Mitochondrial control of apoptosis. Immunol Today 18: 44-51, 1997.

18. Chung TW, Kim JR, Suh JI, Lee YC, Chang YC, Chung TH and $\mathrm{Kim} \mathrm{CH}$ : Correlation between plasma levels of matrix metalloproteinase (MMP)-9/MMP-2 ratio and alpha-fetoproteins in chronic hepatitis carrying hepatitis B virus. J Gastroen Hepatol 19: 565-571, 2004.

19. Lichtinghagen R, Huegel O, Seifert T et al: Expression of matrix metalloproteinase- 2 and -9 and their inhibitors in peripheral blood cells of patients with chronic hepatitis C. Clin Chem 46 183-192, 2000.

20. Chen JS, Wang Q, Fu XH, Huang XH, Chen XL, Cao LQ, Chen LZ, Tan HX, Li W, Bi J and Zhang LJ: Involvement of $\mathrm{PI} 3 \mathrm{~K} / \mathrm{PTEN} / \mathrm{AKT} / \mathrm{mTOR}$ pathway in invasion and metastasis in hepatocellular carcinoma: Association with MMP-9. Hepatol Res 39: 177-186, 2009. 